Michelle R. Garfinkel ${ }^{1}$ / Constantinos Syropoulos ${ }^{2}$

\title{
Rules for Dividing a Disputed Resource in the Context of the Classical Liberal Argument for Peace
}

\author{
${ }^{1}$ Department of Economics, University of California, Irvine, 92697-5100, USA, E-mail: mrgarfin@uci.edu \\ ${ }^{2}$ School of Economics, LeBow College of Business, Drexel University, Philadelphia, PA 19104, USA
}

\begin{abstract}
:
In this paper, we study alternative forms of conflict resolution, both peaceful and non-peaceful, between two countries that compete for claims to a resource used to produce potentially traded goods. Consistent with the classical liberal argument, peace supports mutually beneficial trade, whereas war preempts it. War always induces countries to allocate resources into non-contractible arming ("guns") for superiority in conflict. Under peaceful settlement, countries might choose to arm as well for gaining leverage in negotiations, but arming is typically less than what it is under war. Building on the observation that arming itself affects the countries' bargaining sets, we compare the efficiency properties of division rules generated by three prominent bargaining solutions - namely, splitting the surplus, equal sacrifice, and Nash bargaining - and show how they depend on the gains from trade.
\end{abstract}

Keywords: resource disputes, gains from trade, negotiated settlements, variable threats, endogenous bargaining set

JEL classification: C72, C78, D30, D74, F51

DOI: 10.1515/peps-2017-0036

\section{Introduction}

The longstanding classical liberal view in the international relations literature holds that globalization and/or trade liberalization promotes global peace (e.g. Polachek, 1980). Specifically, to the extent that war between nations disrupts trade between them as Glick and Taylor's (2010) evidence suggests, greater economic interdependence increases the costs of war and thus should decrease the likelihood of its onset. This view finds some support empirically; overall, however, the evidence is mixed. ${ }^{1}$ Moreover, the particular mechanisms underlying this link are not fully understood. Existing theoretical work shows that whether the presence of trading opportunities makes conflict more or less severe (as measured by resources diverted to the military) depends on a number of factors that drive trade and its possible gains, including the source of comparative advantage and the countries' relative sizes. ${ }^{2}$ Even less understood are how different methods of conflict resolution between nations, who possibly trade with each other, affect the potential gains they could realize by settling their differences peacefully.

An obvious advantage of not resorting to war, in addition to leaving open the possibility of trade, is that it allows the two sides to avoid war's destructive effects on productive resources. A related but perhaps less obvious advantage derives from the anticipation that the dispute will be resolved peacefully - namely, to free up resources that otherwise would have to be devoted to building one's military, thereby leaving more for production and trade. In the context of international relations where contracting between countries is incomplete, the magnitude of this benefit would be moderated by the countries' perception that building one's military force in advance of their negotiations, even if not deployed, is necessary to improve one's bargaining position and, thus, obtain a better "deal." If building military force is seen as being more important this way, the possible net gains from a peaceful settlement are smaller; and, conversely, if building such force is seen as being relatively unimportant, the possible net gains are larger. Hence, the conventions or norms of negotiation that spell out how the two sides of a dispute reach an agreement matter for determining the gains that can be realized under a peaceful settlement over war. ${ }^{3}$

In this paper, we explore how such norms interact with the gains from trade possible under peaceful settlement. More specifically, based on a simplified version of the model presented in Garfinkel and Syropoulos 
(2017b) of resource disputes and trade between two countries, we compare the efficiency properties of three different rules of division under settlement. These rules correspond to three prominent bargaining solutions - namely, splitting the surplus, equal sacrifice and Nash bargaining. ${ }^{4}$ They differ in terms of the role assigned to the threat points or payoffs under war, as defined by the quantity "arms" or "guns" brought by each side to the negotiation table. Specifically, the split-the-surplus solution involves an equal division of the difference between the sum of the two sides' payoffs under the agreement and the sum of their threat-point payoffs; the equal sacrifice solution requires that each side's sacrifice in payoffs, defined as the difference between one's maximum feasible payoff (or ideal payoff) and the payoff under the agreed upon division, be equalized; and, the Nash bargaining solution involves maximizing the product the two sides' surpluses realized under the agreement relative to their respective threat points. The different roles assigned to the threat points mean that the rules induce different incentives for arming in anticipation of settlement, which imply different quantities of the resource remaining for production and trade. Therefore, like Anbarci, Skaperdas, and Syropoulos (2002), we find the relative efficiency of the rules depends, at least in part, on their relative sensitivity to the threat points or payoffs under war. As in that paper, variation in such sensitivity across rules can be interpreted as variation in the norms against threats, with greater sensitivity implying weaker norms against threats, greater equilibrium arming and thus greater inefficiency.

Moving beyond Anbarci, Skaperdas, and Syropoulos (2002), however, we find that the shape and size of the bargaining set also depend on the potential gains from trade. ${ }^{5}$ If the gains from trade are large, then at least one of the division rules considered (either that which follows from the equal sacrifice solution or that which follows from split the surplus solution) can support peace with no arming at all; and, in this case, Nash bargaining, which always induces positive arming, is dominated by the other bargaining protocols. However, if the gains from trade are sufficiently small, then all of the bargaining solutions considered involve strictly positive arming; in this case, equal sacrifice - the solution concept that relies least on the disagreement payoffs - is Pareto dominant.

How do the payoffs under peaceful settlement compare with the payoffs under conflict? Under the premise of the classical liberal hypothesis that war disrupts trade, each of the rules considered under peaceful settlement delivers a higher payoff than the payoff under war. What's more, whether the gains from trade are large or small, each of the rules considered supports peaceful settlement as the unique equilibrium outcome. Put differently, provided that there are some gains from trade, war is not a possible equilibrium outcome. ${ }^{6}$

The remainder of the paper proceeds as follows. In the next section, we present a simple one-period model of conflict and trade, along with a preliminary analysis of outcomes and payoffs under war and under peaceful settlement for any division rule considered and given the gun choices made by each side of conflict. In Section 3 , we turn to study each of the rules of division implied by the three bargaining solutions and derive our central findings. Section 4 offers concluding remarks, including possible extensions of the analysis. Technical details are relegated to Appendix A.

\section{A simple model of conflict and trade}

Consider an environment in which each of two agents/countries, $i=1,2$, is endowed with $R^{i}$ units of a productive resource such as land, oil or water. Once held securely, this resource can be used to produce (possibly tradable) commodities. However, initial claims are not secure. Each agent invests $G^{i}$ units of $R^{i}$ in a non-contractible and irreversible influence activity called "arming" (or "guns" for short) to contest the remaining resource jointly held by both: $\bar{X} \equiv \sum_{i}\left(R^{i}-G^{i}\right)$.

Their competing claims over $\bar{X}$, given their prior arming choices, can be resolved in one of two ways: through a winner-take-all contest ("war") or through bargaining and negotiation ("peaceful settlement"). Under war which precludes trade, each agent's probability of winning the contest depends on the agents' relative arming. ${ }^{7}$ Under peaceful settlement, the agents divide $\bar{X}$ on the basis of some predetermined bargaining solution concept. Once $\bar{X}$ is divided and the agents produce their goods, they engage in free trade with each other.

A distinguishing feature of peaceful settlement in this setting is that it is conducted under the threat of war. That is to say, if the two agents reach an impasse in their negotiations, war breaks out, with each agent's probability of winning all of $\bar{X}$ depending on his/her relative guns. As will become evident, arming provides, through its impact on the disagreement or war payoffs, agents with leverage in their negotiations. But, by diverting resources away from useful production, arming also affects (i) the size of the "prize" and (ii) the trade-off in payoffs along the payoff possibility frontier. To the extent that division rules differ in their reliance on the just noted dimensions of the bargaining problem, their efficiency properties could differ as well. The question is: How? 


\subsection{Production and trade for given effective endowments}

We now turn to production and trade that are important for characterizing the bargaining set and thus for answering the question above. Let the share of $\bar{X}$ obtained by agent $i$ under peaceful settlement based on the solution concept $h \in\{s s, e s, n b\}$ be denoted by $\lambda_{h}^{i}$, where "ss" is identified with splitting the surplus, "es" with

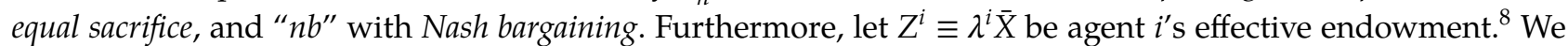
assume for simplicity that $Z^{i}$ can be used to produce, on a one-to-one basis, a nationally differentiated and potentially tradable commodity. We interpret this commodity as an intermediate input to the production of a final consumption good, say "butter."

To capture the gains from trade in a compact and intuitive way, we assume the production function for butter takes the constant elasticity of substitution (CES) form. Production by agent $i$ is given by

$$
F^{i}=F\left(D_{1}^{i}, D_{2}^{i}\right)=\left[\left(D_{1}^{i}\right)^{\frac{\sigma-1}{\sigma}}+\left(D_{2}^{i}\right)^{\frac{\sigma-1}{\sigma}}\right]^{\frac{\sigma}{\sigma-1}},
$$

where $D_{j}^{i}$ denotes the quantity of intermediate input $j \in\{1,2\}$ demanded by agent $i \in\{1,2\}$, and $\sigma>1$ denotes the elasticity of substitution between the two intermediate inputs. The specification in (1), which is symmetric, increasing, linearly homogeneous, and (provided $\sigma<\infty$ ) strictly concave in its arguments, reflects the benefit of employing a greater variety of distinct inputs in production, analogous to the "love of variety" exhibited by Dixit-Stiglitz preferences; and, this benefit, which can be interpreted as the gains from trade, increases as the two intermediate goods become more distinct (i.e. as $\sigma(>1)$ falls). ${ }^{10}$

Whether we consider trade or autarky, agent $i$ chooses the allocation of his effective endowment $Z^{i}$ to maximize his output and thus consumption of butter $F^{i}$ shown in (1). Of course, the constraints relevant for that choice depend on whether the two agents trade or not. Under autarky, each agent $i$ is forced to rely solely on his own productive capabilities; thus, $D_{j}^{i}=0$ for $i \neq j$ and $F^{i}=F(\cdot)=Z^{i}$ in this case.

The case of free trade is a bit more involved. Let $Y^{i}, p_{j}^{i}$ and $\gamma_{j}^{i}$ respectively denote agent $i^{\prime}$ s income, price and expenditure share on good $j$. One can verify that $\gamma_{j}^{i} \equiv\left(p_{j}^{i} / P^{i}\right)^{1-\sigma}$, where $P^{i} \equiv\left[\sum_{j}\left(p_{j}^{i}\right)^{1-\sigma}\right]^{\frac{1}{1-\sigma}}$, and furthermore that $D_{j}^{i}=\gamma_{j}^{i} Y^{i} / p_{j}^{i}$, where $Y^{i}=p_{i}^{i} Z^{i}$. Now let the "world" price of good $j$ be denoted by $\pi_{j}$. Our assumption of free trade implies $p_{j}^{i}=\pi_{j}$ for each agent $i=1,2$ and both goods $j=1,2$. Naturally, relative prices are endogenously determined. By Walras' Law, these prices follow from the world market-clearing condition $\pi_{j} D_{j}^{i}=\pi_{i} D_{i}^{j}(i \neq j)$, and satisfy

$$
\pi_{T} \equiv \pi_{j} / \pi_{i}=\left(Z^{i} / Z^{j}\right)^{1 / \sigma}=\left(\lambda^{i} / \lambda^{j}\right)^{1 / \sigma},
$$

where the far right expression was obtained from the definition $Z^{i} \equiv \lambda^{i} \bar{X}$.

\subsection{Payoffs under peaceful settlement}

To derive an expression for agent $i$ 's payoff function under trade presuming peaceful negotiations are successful $\left(V^{i}=F^{i}\right)$, we combine (2) and the relations noted above with (1) to obtain, after simplification,

$$
V^{i} \equiv V^{i}\left(\lambda^{i}, G^{i}, G^{j}\right)=v^{i} \bar{X}
$$

where

$$
v^{i}\left(\lambda^{i}\right) \equiv \lambda^{i}\left[1+\left(\lambda^{i} / \lambda^{j}\right)^{\frac{1-\sigma}{\sigma}}\right]^{\frac{1}{\sigma-1}}=\left[\frac{\left(\lambda^{i}\right)^{\frac{\sigma-1}{\sigma}}}{\left(\lambda^{i}\right)^{\frac{\sigma-1}{\sigma}}+\left(\lambda^{j}\right)^{\frac{\sigma-1}{\sigma}}}\right] F\left(\lambda^{i}, \lambda^{j}\right) .
$$

The far right expression in (4) reveals that the payoff to agent $i$, per unit of the contested resource $\bar{X}$, coincides with his competitive share of the output that would be produced in an integrated competitive economy. ${ }^{11}$ That is to say, each agent is rewarded with the value of his marginal product times the quantity of the resource he controls.

Since as one can easily confirm $\arg \max _{\lambda^{i}} F\left(\lambda^{i}, \lambda^{j}\right)=\frac{1}{2}$ and $v^{i}+v^{j}=F\left(\lambda^{i}, \lambda^{j}\right)$, each agent would be willing to sacrifice some efficiency (i.e. a reduction in $\left.F\left(\lambda^{i}, \lambda^{j}\right)\right)$ in return for a larger share $\lambda^{i}$ of the contested resource. It is 
straightforward to verify that, provided the two intermediate inputs are distinct (i.e. $\sigma<\infty), v^{i}$ is strictly concave in $\lambda^{i}$, that $\lambda_{T}^{i} \equiv \arg \max _{\lambda^{i}} v^{i} \in\left(\frac{1}{2}, 1\right)$ and that $\lim _{\sigma \rightarrow 1} \lambda_{T}^{i}=\frac{1}{2}$, while $\lim _{\sigma \rightarrow \infty} \lambda_{T}^{i}=1 .{ }^{12}$ Clearly, the elasticity of substitution $\sigma$ plays a key role in the characterization of the critical values of $\lambda^{i}$ for payoffs under trade. Indeed, as we will see shortly, the value of $\sigma$ figures prominently in the determination of the bargaining set and the ranking of payoffs that the division rules $h \in\{s s, e s, n b\}$ deliver.

\subsection{Payoffs under war}

Turning to war, let $\phi^{i}\left(G^{i}, G^{j}\right)$ be the probability that agent $i(\neq j)$ wins. For simplicity, we assume the ratio form of the conflict technology:

$$
\phi^{i}\left(G^{i}, G^{j}\right)=\left\{\begin{array}{lll}
\frac{G^{i}}{G^{i}+G^{j}}, & \text { if } & G^{i}+G^{j}>0 \\
\frac{1}{2}, & \text { if } & G^{i}+G^{j}=0 .
\end{array}\right.
$$

According to this specification, agent $i$ 's winning probability is increasing in his own guns $G^{i}$, decreasing in his rival's guns $G^{j}$ and is symmetric so that $\phi^{i}=\phi^{j}=\frac{1}{2}$ when $G^{i}=G^{j} .^{13}$

Recall that, since there is no trade under war, the expression in (1) implies that production depends linearly on the agent's own effective endowment: $F^{i}=Z^{i}$. Now let $Z_{W} \equiv \bar{X}$ denote the effective endowment enjoyed by the winner of the conflict and $Z_{L}=0$ denote the loser's effective endowment. Then, we can write agent $i$ 's expected payoff under conflict, denoted by $U^{i}$, as

$$
U^{i} \equiv U^{i}\left(G^{i}, G^{j}\right)=\phi^{i} Z_{W}^{i}+\left(1-\phi^{i}\right) Z_{L}^{i}=\phi^{i} \bar{X} .
$$

For future reference, define $u^{i} \equiv \phi^{i}$, so that $u^{i}+u^{j}=1$ and $U^{i}=u^{i} \bar{X}$. As one can confirm, by differentiating the far right expression in (6) with respect to $G^{i}$, recalling $\bar{X}=\sum_{i}\left(R^{i}-G^{i}\right)$, using (5) and examining the resulting first-order conditions (FOCs), agent $i^{\prime}$ s best response to agent $j^{\prime}$ s choice $G^{j}, B_{c}^{i}\left(G^{j}\right)$, is given by

$$
B_{c}^{i}\left(G^{j}\right)=\max \left[R^{i}, \sqrt{G^{j}\left(R^{i}+R^{j}\right)}-G^{j}\right],
$$

which implies a symmetric equilibrium under war, $G_{c}^{i}=G_{c}=\frac{1}{4}\left(R^{i}+R^{j}\right)$ for $i=1$, 2, when neither agent is resource constrained in his arming choice. ${ }^{14}$

\subsection{A comparison of payoffs for given guns}

To understand the nature of the bargaining protocols we wish to study - and, in particular, the way in which they condition arming incentives - it helps to fix guns at some level and ask how the per unit of $\bar{X}$ payoff vectors, $\left(v^{i}, v^{j}\right)$ under settlement and $\left(u^{i}, u^{j}\right)$ under conflict, compare. As suggested earlier, the elasticity of substitution $\sigma$ is a crucial parameter here. Figure 1 illustrates the dependence of the above payoffs (per unit of $\bar{X}$ ) along the payoff possibility frontier (PPF) for several values of $\sigma$. The black, solid-line segment that connects point 1 on the two axes depicts the possible payoff values under conflict, $u^{i}$. Points along this schedule define the agents' disagreement payoffs which, of course, depend on their relative arming. The midpoint of this schedule depicts the combination of the agents' expected payoffs under war that arises when $G^{1}=G^{2}$. In the extreme case, where $\sigma \rightarrow \infty$ such that the intermediate tradables are perfect substitutes, the PPF for $\left(v^{i}, v^{j}\right)$ coincides with the $u^{1}+u^{2}$ $=1$ frontier; in this special case, trade and, thus, settlement are pointless. 

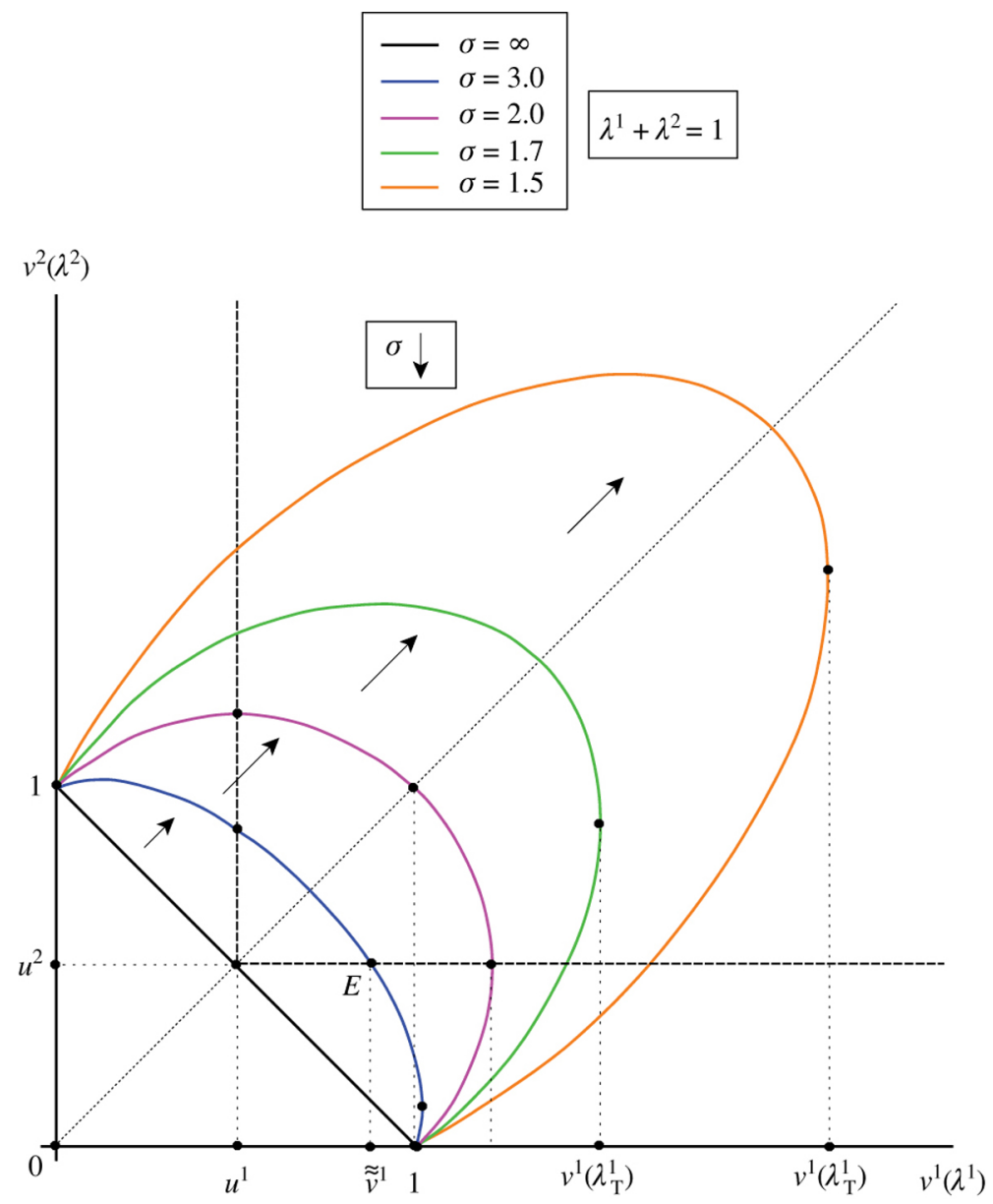

Figure 1: Bargaining Sets and their Dependence on the Gains from Trade.

Now suppose $\sigma$ is finite, which implies as noted earlier that $v^{i}(\cdot)$ is strictly concave in $\lambda^{i}$. Then, for any feasible pair of guns $\left(G^{i}, G^{j}\right)$, there exist many combinations of shares, $\lambda^{i}$ and $\lambda^{j}=1-\lambda^{i}$, for which both agents are weakly better off under settlement $\left(v^{i} \geq u^{i}\right.$ for $\left.i=1,2\right)$, and at least one agent is strictly better off $\left(v^{i}>u^{i}\right)$. The collection of such combinations gives us the bargaining set. Figure 1 depicts this set, in the symmetric case where $G^{i}=$ $G^{j}$, for alternative (finite) values of $\sigma \cdot{ }^{15}$ Importantly, the lower is the value of $\sigma(>1)$ and thus the greater are the possible gains from trade, the larger are the potential gains from settlement and thus the larger is the size of the bargaining set. The particular rule of division used by the two agents, then, pins down a point on the relevant PPF. For example, a division of the contested resource according to $\phi^{i}$ given $G^{i}=G^{j}$ would pick the $\left(v^{i}, v^{j}\right)$ combination where the $45^{\circ}$ line intersects the relevant PPF (see Figure 1). More generally, the strict concavity of $v^{i}(\cdot)$ implies, again for given $\left(G^{i}, G^{j}\right)$, the two sides always find it appealing to settle their disputes peacefully by dividing the resource $\bar{X}$ according to $\phi^{i}$ instead of initiating war and winning all of $\bar{X}$ with probability $\phi^{i}$.

But, while the division of the resource according to $\phi^{i}$ is one possibility, other division rules, such as the ones noted earlier, could be considered. To prepare the groundwork for this analysis, we start with the definition of an agent's "ideal" payoff. Let $\tilde{\lambda}^{j}$ denote the smallest share of the contested resource that agent $j$ would be willing to accept in a peaceful settlement. This share satisfies

$$
\tilde{v}^{j} \equiv v^{j}\left(\tilde{\lambda}^{j}\right)=u^{j}(\cdot),
$$

with $u^{j}=\phi^{j}$ defined in (5) and $v^{j}$ defined in (4). This share for agent $j$ would leave agent $i$ with a share $\tilde{\tilde{\lambda}}^{i}=1-\tilde{\lambda}^{j}$ that generates a payoff

$$
\tilde{\tilde{v}}^{i} \equiv v^{i}\left(\tilde{\tilde{\lambda}}^{i}\right) .
$$

This payoff combination is illustrated in Figure 1, assuming $G^{i}=G^{j}$ and $\sigma=3$, at point $E$. However, depending on the degree of substitutability between the two inputs $(\sigma), \lambda_{T}^{i} \equiv \arg \max _{\lambda^{i}} v^{i}$ could be less than $\tilde{\tilde{\lambda}}^{i}$, in which case agent $i$ would obtain a higher payoff by accepting a smaller share of the contested resource - i.e. $v^{i}\left(\lambda_{T}^{i}\right)>v^{i}\left(\tilde{\lambda}^{i}\right)$ 
- which his rival would gladly accept. This reasoning suggests that an agent's "ideal" payoff in the bargaining set (denoted by $w^{i}$ ) can be defined as

$$
w^{i}=\max \left[v^{i}\left(\tilde{\lambda}^{i}\right), v^{i}\left(\lambda_{T}^{i}\right)\right] .
$$

The next lemma builds on these ideas to characterize some important properties of the payoffs $v^{i}$ and $w^{i}$ as they depend on the potential gains from trade:

\section{Lemma 1}

Under settlement, agent $i$ 's payoff $v^{i}$ and ideal payoff $w^{i}$ for $i=1,2$ satisfy the following relationships:

1. If $\sigma \lesseqgtr 2$, then $v^{i}\left(\frac{1}{2}\right) \gtreqless v^{i}(1)$.

2. Suppose $G^{1}=G^{2}=G$. Then, $w^{i}=v^{i}\left(\lambda_{T}^{i}\right)$ for $\sigma \leq 2$ and $w^{i}=v^{i}\left(\tilde{\tilde{\lambda}}^{i}\right)$ for $\sigma \geq 2$.

Proof: See Appendix A.

Part (1) suggests a potential trade-off between ownership and trade. In particular, it establishes that, when the gains from trade are sufficiently large $(\sigma<2)$, both agents prefer to share the disputed resource equally and then engage in trade over controlling the entire resource in which case trade would not be possible. Otherwise (when $\sigma \geq 2$ ), both agents prefer to control the entire resource. ${ }^{16}$ Part (2) shows that, when both agents produce identical quantities of guns and the gains from trade are relatively large $(\sigma \leq 2)$, each agent's ideal payoff coincides with the payoff he would enjoy if he were free to choose the division of $\bar{X}$ solely on the basis of self interest, as indicated by $\lambda_{T}^{i}$. By contrast, when $\sigma \geq 2$, an agent's ideal payoff depends on the disagreement payoffs, as indicated by $\tilde{\tilde{\lambda}}^{i}$ that gives his rival the lowest acceptable payoff. Figure 1 illustrates these points.

\section{Division rules and their relative efficiency}

We now study the efficiency properties of three division rules generated by the three bargaining protocols identified earlier - namely, the split-the-surplus (ss), equal sacrifice (es) and Nash bargaining $(n b)$ solutions. After formally defining these rules, we compare their implications for arming and payoffs and ultimately for the relative appeal of peace. Henceforth, we adopt the assumption of symmetry across agents/countries:

$$
\text { Symmetry: } R^{i}=R \text { for } i=1,2 \text {. }
$$

Under this assumption, equilibrium spending under peaceful settlement for any rule $h \in\{s s, e s, n b\}$ will be symmetric.

As noted earlier, $\lambda_{h}^{i}$ (for $i=1,2$ and $h \in\{s s, e s, n b\}$ ) denotes the share of the common pool obtained by agent $i$ under solution $h$. We similarly index payoffs $v_{h}^{i}=v^{i}\left(\lambda_{h}^{i}\right)$ and $V_{h}^{i}=V^{i}\left(\lambda_{h}^{i}\right)\left(=v_{h}^{i} \bar{X}\right)$. Keeping guns (and thus $\bar{X})$ fixed in the background, define the following functional relationships:

$$
\begin{gathered}
\Phi \equiv \Phi\left(\lambda^{i}, G^{i}, G^{j}\right)=\frac{v^{j}-u^{j}}{v^{i}-u^{i}} \\
\Psi \equiv \Psi\left(\lambda^{i}, G^{i}, G^{j}\right)=\frac{w^{j}-v^{j}}{w^{i}-v^{i}} \\
\Omega \equiv \Omega\left(\lambda^{i}\right)=-\frac{v_{\lambda^{j}}^{j}}{v_{\lambda^{i}}^{i}}
\end{gathered}
$$

for $i \neq j=1$, 2, where we use the convention that $v_{\lambda^{i}}^{i} \equiv d v^{i} / d \lambda^{i}-d v^{i} / d \lambda^{j}$ (since $\lambda^{j}=1-\lambda^{i}$ ). Then, the solution concepts noted above can be defined as follows:

$$
\Phi\left(\lambda^{i}, G^{i}, G^{j}\right)=1 \Rightarrow \frac{v^{j}\left(\lambda_{s s}^{j}\right)-u^{j}}{v^{i}\left(\lambda_{s s}^{i}\right)-u^{i}}=1
$$




$$
\begin{gathered}
\Psi\left(\lambda^{i}, G^{i}, G^{j}\right)=1 \Rightarrow \frac{w^{j}-v^{j}\left(\lambda_{e s}^{j}\right)}{w^{i}-v^{i}\left(\lambda_{e s}^{i}\right)}=1 \\
\Phi\left(\lambda^{i}, G^{i}, G^{j}\right)+\Omega\left(\lambda^{i}\right)=0 \Rightarrow \frac{v^{j}\left(\lambda_{n b}^{j}\right)-u^{j}}{v^{i}\left(\lambda_{n b}^{i}\right)-u^{i}}=\frac{v_{\lambda^{j}}^{j}\left(\lambda_{n b}^{j}\right)}{v_{\lambda^{i}}^{i}\left(\lambda_{n b}^{i}\right)} .
\end{gathered}
$$

Since $\lambda_{h}^{j}=1-\lambda_{h}^{i}$, the shares $\lambda_{h}^{i}$ induced by the various solution concepts are implicitly defined by the above equations.

These concepts can be visualized with the help of the two panels in Figure 2, drawn for given arms with $G^{1}>$ $G^{2} \cdot{ }^{17}$ In both panels, the combination of payoffs $v_{h}^{1}$ and $v_{h}^{2}$ under each of the solutions considered $h \in\{s s, e s, n b\}$ lie on the PPF. The ideal payoff for agent $1 w^{1}$ is defined by $\lambda_{T}^{1}$ in panel (A) where $\sigma<2$ and is defined by $\tilde{\lambda}^{1}$ in panel (B) where $\sigma>2$. For the rule generated by $s s, \lambda_{s s}^{i}$ is implicitly defined (by (15)) as point $s s$ in the panels, because $\Phi=1$ holds along the $45^{\circ}$ line going through the $\left(u^{1}, u^{2}\right)$ point of disagreement payoffs. Similarly, the $\lambda_{e s}^{i}$ share is defined (by (16)) as point $e$ in both panels, because $\Psi=1$ holds along the $45^{\circ}$ line going through the point of ideal payoffs $\left(w^{1}, w^{2}\right)$. Finally, the Nash bargaining solution satisfies $\left.\lambda_{n b}^{i}=\arg \max _{\lambda^{i}}\left[v^{i}\left(\lambda^{i}\right)-u^{i}\right)\right]\left[v^{j}\left(\lambda^{j}\right)-u^{j}\right]$. The expression in (17) represents the FOC to this problem.

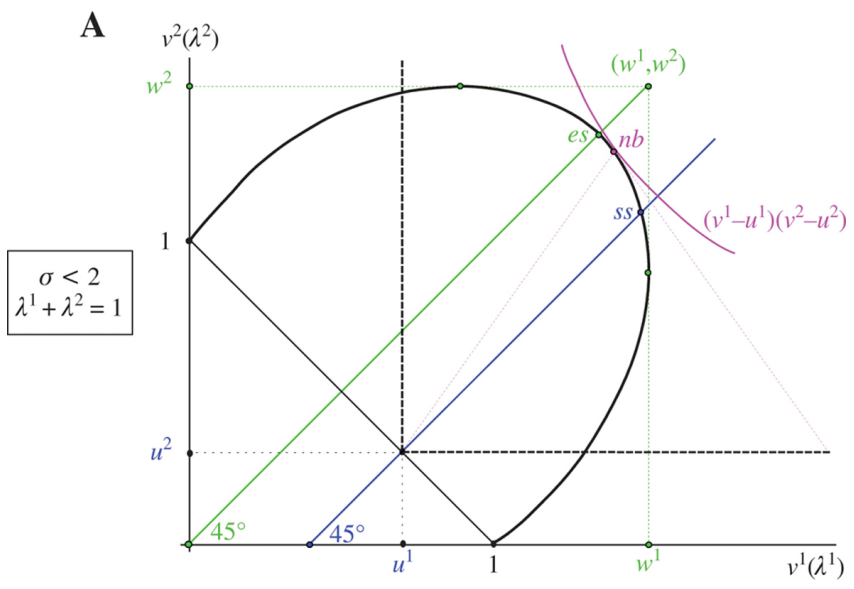

B

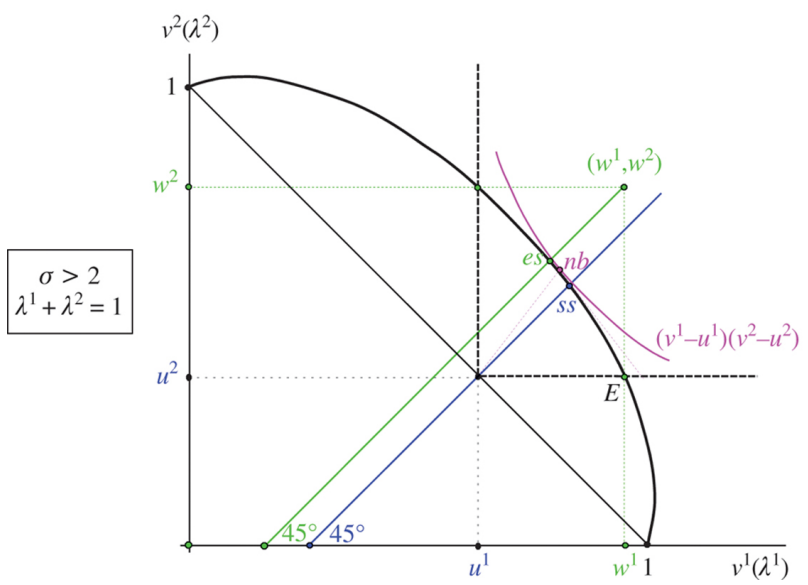

Figure 2: Disagreement Payoffs, Ideal Payoffs and Division Rules.

It is important to emphasize that changes in guns do not directly affect payoffs $v_{h}^{i}$. However, changes in guns can affect shares (and thus the payoffs $v_{h}^{i}$ ) through their impact on the disagreement payoffs $\left(u^{i}, u^{j}\right)$. In the case of equal sacrifice, the effect of guns on $\lambda_{e s}^{i}$ is transmitted through their impact on the ideal payoffs which, as we saw earlier, can depend on guns indirectly (though not always), again through the disagreement payoffs.

To identify the impact of guns on payoffs we partially differentiate $V_{h}^{i}=v_{h}^{i} \bar{X}$ with respect to $G^{i}$, accounting for the effect on the resource that remains, $\bar{X}$ : 


$$
\partial V_{h}^{i} / \partial G^{i}=\bar{X}\left(\frac{\partial v_{h}^{i}}{\partial \lambda_{h}^{i}}\right)\left(\partial \lambda_{h}^{i} / \partial G^{i}\right)-v_{h^{\prime}}^{i} i=1,2 \text { and } h \in\{s s, e s, n b\} .
$$

The first term represents the indirect effect of an additional gun on agent $i$ 's payoff through its effect on the division rule; the second term captures the constant marginal cost as an additional gun implies a smaller quantity of the resource $\bar{X}$ to divide. It should now be evident that the sensitivity $\partial \lambda_{h}^{i} / \partial G^{i}$ of an agent's share to his guns is central to his arming decision.

The next lemma describes this sensitivity across the three solution concepts:

\section{Lemma 2}

Assume symmetry (11) holds and suppose that $\lambda_{h}^{i}$ responds to changes in $G^{i}(i=1,2$ and $h \in\{s s, e s, n b\})$. Then, $0<\partial \lambda_{e s}^{i} / \partial G^{i}<\partial \lambda_{n b}^{i} / \partial G^{i}<\partial \lambda_{s s}^{i} / \partial G^{i}$, where each partial derivative is evaluated at an identical quantity of arms $G^{i}=$ $G^{j}=G$

Proof: See Appendix A.

Maintaining (momentarily) a focus on situations where agents' shares $\lambda_{h}^{i}$ respond to changes in arming, Lemma 2 points out that, under conditions of symmetry, equal sacrifice gives rise to a division that is least sensitive to an agent's arms, whereas splitting the surplus gives rise to a division that is most sensitive to an agent's arms; the sensitivity of the division to an agent's arms under Nash bargaining lies in the middle. Although Anbarci, Skaperdas, and Syropoulos (2002) do not consider Nash bargaining, they find a similar ranking for the equal sacrifice and split-the-surplus solutions in a different setting. But, the more important point here is this: the bargaining solution that relies least (most) on the threat-point payoffs corresponds precisely to the division that is least (most) sensitive to changes in arming. Interestingly, the Nash bargaining solution lies in the middle of this ranking because, even though it relies on the disagreement payoffs, this reliance is tempered by its dependence on the slope of the PPF and the sensitivity of that slope to arming.

The above discussion raises the question of whether, under our assumption of symmetry, each of the bargaining solutions $h \in\{s s, e s, n b\}$ necessarily implies a rule of division $\lambda_{h}^{i}$ that depends positively on agent $i$ 's arming given the rival's arming. As we now argue, the presence or absence of such an influence of guns depends on the magnitude of the gains from trade (which depends negatively on $\sigma>1$ ) as well the bargaining solution considered.

\section{Proposition 1}

(Symmetric equilibria.) Assume (11), let $G_{h}$ denote the representative agent's guns in the symmetric equilibria under each bargaining solution $h \in\{s s, e s, n b\}$, and define $A \equiv 2^{\frac{1}{\sigma-1}} \frac{(\sigma-1)}{\sigma}(>0)$ and $B \equiv \frac{\frac{1}{\sigma-1}-1}{\sigma-1}(>0)$. Then,

1. under the split-the-surplus protocol, equilibrium arming satisfies $G_{s s}=0$ and/or $G_{s s}=R /\left[1+\frac{\sigma}{\sigma-1} A\right]$ :

a. if $\sigma \in\left[1 \frac{1}{2}, 2\right]$, there exist two equilibria one of which exhibits no arming;

b. if $\sigma \in\left(1,1 \frac{1}{2}\right) \cup(2, \infty)$, there exists a unique equilibrium with positive arming.

2. under the equal sacrifice and Nash bargaining protocols, there exist unique equilibria such that

a. $G_{e s}=0$ for $\sigma \in(1,2]$, while $G_{e s}>0$ for $\sigma \in(2, \infty)$;

b. $G_{n b}=R /\left[1+\frac{\sigma}{\sigma-1}(A+B)\right]$ for $\sigma \in(1, \infty)$.

Proof: See Appendix A.

Four ideas stand out in connection with Proposition 1. First, under the split-the-surplus solution and depending on the size of the gains from trade, two types of (symmetric) equilibria can arise: one with and another without arming. As explained in Garfinkel and Syropoulos (2017b), this possibility arises in a more general setting and can be explained as follows. For all $\sigma \in(1, \infty]$, given the rival's arming is strictly positive, each agent $i$ has a strictly positive incentive to arm as well under the split-the-surplus solution. However, when $\sigma$ $<2$, the split-the-surplus rule allocates a strictly positive share of the resource to the rival even when he does not arm and agent $i$ does. Now suppose that $\sigma$ is marginally below 2 and $G^{j}=0$. Agent $i$ can choose $G^{i}=\varepsilon$ (an infinitesimal quantity) and obtain a share close to 1 or choose $G^{i}=0$ and obtain half of $\bar{X}$. By part (1) of Lemma 1, agent $i$ prefers the latter. Nevertheless, as $\sigma$ falls, the relative appeal of choosing $G^{i}=0$ when $G^{j}=$ 0 eventually diminishes and approaches zero as $\sigma$ approaches $\frac{3}{2}$, thereby undermining the stability of $G_{s s}=0$ 
for $\sigma \in\left(1, \frac{3}{2}\right) .{ }^{18}$ Second, the symmetric equilibria under the equal sacrifice and Nash bargaining solutions are unique. The reason is that these solutions, unlike the split-the-surplus solution, do not set in motion a process that leads to no arming. Third, the equilibrium under Nash bargaining always involves a positive quantity of guns. In contrast, the equilibrium under equal sacrifice involves no arming when $\sigma \leq 2$ and positive arming when $\sigma>2$. The reason $G_{n b}>0$ is because the solution is always at a negatively sloped portion of the PPF and its implementation requires each agent to produce some guns. The reason $G_{e s}=0$ for $\sigma \leq 2$ is because in this case agents' ideal payoffs are independent of the disagreement payoffs, as emphasized in part (2) of Lemma 1 , and, consequently, are invariant to changes in arms. By contrast, when $\sigma>2, G_{e s}>0$ because agents' ideal payoffs depend on $\left(u^{1}, u^{2}\right)$ and thus on arming decisions. Finally, in interior equilibria, the quantity of arms under splitting the surplus is larger than that under Nash bargaining. This ranking, which can be deduced from the equilibrium quantities described in the proposition, is driven by the ranking of the sensitivities of shares detailed in Lemma 2.

The next proposition, which follows from Proposition 1 with Lemma 1 and Lemma 2, provides a more precise statement of these ideas.

\section{Proposition 2}

(Arming Comparisons.) Under the assumption of symmetry (11), the equilibrium quantities of guns associated with the bargaining solutions $h \in\{s s, e s, n b\}$ can be compared as follows:

1. If $\sigma \in\left(1,1 \frac{1}{2}\right)$, then $G_{e s}=0<G_{n b}<G_{s s}$.

2. If $\sigma \in\left(1 \frac{1}{2}, 2\right]$, then $G_{e s}=G_{s s}=0<G_{n b}$ or $G_{e s}=0<G_{n b}<G_{s s}$.

3. If $\sigma \in(2, \infty)$, then $0<G_{e s}<G_{n b}<G_{s s}$.

The horizontal axis in Figure 3 plots the equilibrium level of arming associated with each solution concept under peaceful settlement and that under war. Panel (A) considers a case where $\sigma \in\left(1 \frac{1}{2}, 2\right]$ (as in part (2) of the proposition), and panel (B) considers a case where $\sigma>2$ (as in part (3)). One can also verify easily, as shown in the figure, peaceful settlement with any rule $h \in\{s s, e s, n b\}$ induces lower equilibrium arming than what emerges under conflict for all $\sigma \in(1, \infty)$ under assumption (11). ${ }^{19}$

\section{A}

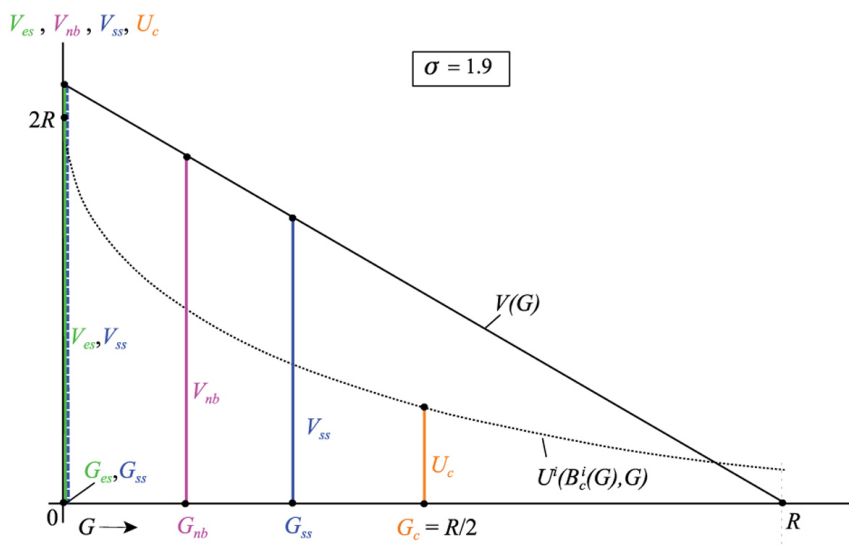

\section{B}

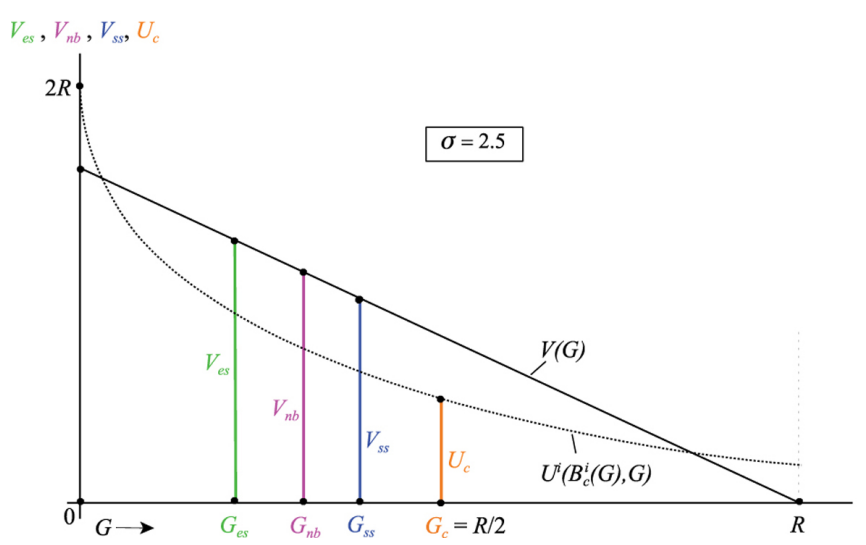


Figure 3: Equilibrium Arming and Payoffs under the Split-the-Surplus, Equal Sacrifice and Nash Bargaining Solutions

It is worthwhile pointing out how the results in Proposition 2 compare with the results in Anbarci, Skaperdas, and Syropoulos (2002). As suggested earlier in connection to Lemma 2, our finding, that for all $\sigma \in(1, \infty)$ equilibrium arming under equal sacrifice is no greater than that under split-the-surplus (i.e. $G_{e s} \leq G_{s S}$ ), is similar to their ranking. Nonetheless, there are two important differences. First, in the setting of that paper with no trade, the ideal payoffs are always sensitive to the threat-point payoffs, while in setting of this paper where there is trade, they need not be - namely, under the equal sacrifice protocol when $\sigma<2$. As such, we find in contrast to Anbarci, Skaperdas, and Syropoulos (2002) that, depending on the magnitude of the possible gains from trade, the rule of division that follows from the equal sacrifice bargaining solution can support unarmed peace. Second, depending on the potential gains from trade, the marginal benefit from arming for agent $i$, under the rule of division that follows from the split-the-surplus solution given $G^{j}=0$, can fall short of the marginal cost. More precisely and in contrast to Anbarci, Skaperdas, and Syropoulos (2002), we find that, when $\sigma \in\left(\frac{3}{2}, 2\right)$, the split-the-surplus protocol can also support unarmed peace. ${ }^{20}$

Proposition 1 and Proposition 2 show how equilibrium arming compares across the three solution concepts. The next step is to compare the associated payoffs. Let $V_{h}$ be the representative agent's payoff when $G^{i}=G_{h}(i=$ $1,2$ and $h \in\{s s, e s, n b\})$. It is easy to see that $V_{h}=2^{\frac{1}{\sigma-1}}\left(R-G_{h}\right)$, which is linearly decreasing in $G_{h}$, as indicated by the downward sloping line in Figure 3. Then, the next proposition follows readily from Proposition 2.

\section{Proposition 3}

(Payoff Comparisons.) Under assumption (11), the equal sacrifice solution weakly dominates the split-the-surplus and Nash bargaining solutions. Furthermore, the Nash bargaining solution dominates the split-the-surplus solutions in equilibria that involve positive quantities of guns. However, this ranking is reversed when the no arming equilibrium under the split-the-surplus solution is considered. More specifically, the equilibrium payoffs associated with the bargaining solutions $h \in\{s s, e s, n b\}$ can be compared as follows:

1. If $\sigma \in\left(1,1 \frac{1}{2}\right)$, then $V_{e s}>V_{n b}>V_{s s}$.

2. If $\sigma \in\left(1 \frac{1}{2}, 2\right]$, then $V_{e s}=V_{s s}>V_{n b}$ for $G_{s s}=0$, while $V_{e s}>V_{n b}>V_{s s}$ for $G_{s s}>0$.

3. If $\sigma \in(2, \infty)$, then $V_{e s}>V_{n b}>V_{s s}$.

Proposition 3 as well as Proposition 2 are illustrated in Figure 3.

Finally, the reader might wonder whether any or all of the equilibria under peaceful settlement based on these division rules are stable. The next proposition addresses this issue.

\section{Proposition 4}

(Stability of equilibrium.) The equilibria under each of the rules of division $h \in\{s s, e s, n b\}$ are immune to unilateral deviations.

The argument here is rather intuitive, and starts with the observation that, for any feasible arming by the two agents $G^{i}$ and $G^{j}$ (not necessarily equal), the payoff for each agent under war is less than the payoff under settlement (and thus trade): $u^{i}\left(G^{i}, G^{j}\right)<v_{h}^{i}\left(G^{i}, G^{j}\right)$ for $i \neq j=1,2$, where we now denote agent $i^{\prime}$ s payoff under war given $G^{i}$ and $G^{j}$ per unit of $\bar{X}$ by $u^{i}\left(G^{i}, G^{j}\right)=\phi^{i}\left(G^{i}, G^{j}\right)$ and agent $i^{\prime}$ s payoff under settlement with division rule $h$ conditional again on $G^{i}$ and $G^{j}$ per unit of $\bar{X}$ by $v_{h}^{i}\left(G^{i}, G^{j}\right)$. But, if that inequality holds for any feasible pair $\left(G^{i}, G^{j}\right)$, then it will surely hold for $\left(B_{c}^{i}\left(G_{h}^{j}\right), G_{h}^{j}\right)$, where $B_{c}^{i}\left(G_{h}^{j}\right)$ indicates agent $i$ 's best reply to the rival's choice, shown in (7), evaluated at $G^{j}=G_{h}^{j}: u^{i}\left(B_{c}^{i}\left(G_{h}^{j}\right), G_{h}^{j}\right)<v_{h}^{i}\left(B_{c}^{i}\left(G_{h}^{j}\right), G_{h}^{j}\right)$. That is to say, even if agent $i$ were to anticipate choosing war and arm accordingly given agent $j^{\prime}$ s arming choice in anticipation of settlement, agent $i$ would be better off choosing settlement over war. But, if agent $i$ anticipates choosing settlement, he would adjust his guns in anticipation of settlement, implying $v_{h}^{i}\left(B_{c}^{i}\left(G_{h}^{j}\right), G_{h}^{j}\right)<v_{h}^{i}\left(G_{h^{\prime}}^{i} G_{h}^{j}\right)$. Thus, unilateral deviations from the settlement outcome under rule $h$ that would trigger a war cannot be profitable. Notice that this argument does not require we impose the assumption of symmetry. Perhaps more importantly, this line of reasoning implies that war cannot emerge as an equilibrium outcome in the one-period setting of this paper. In particular, even if either one or both agents were to arm in anticipation of war, neither would have an incentive to declare war.

\section{Concluding remarks}

The idea that the presence of trade opportunities and the potential gains they imply for participating countries can help promote global peace is by no means new. Indeed, we have shown that, in the setting of our model 
despite its abstraction from war's destructive effects, countries will always prefer a peaceful division of the productive resource followed by mutually beneficial trade over a winner-take-all contest (i.e. war) that precludes trade. But, going beyond that intuitive idea, this paper explores how different rules of division that follow from a variety of bargaining solutions rank in terms of their efficiency. One key result here, as in Anbarci, Skaperdas, and Syropoulos (2002) who do not consider trade, is that the ranking depends on how sensitive the rules of division are to arming through their influence on the disagreement payoffs (or the payoffs obtained under war). The greater is that sensitivity or the weaker are norms against threats, the greater are the incentives to arm under peaceful settlement, leaving fewer resources left over for production.

Building on that idea and extending Anbarci, Skaperdas, and Syropoulos (2002), our analysis shows further that the ranking of the sensitivity of these rules depends on the gains from trade. When the gains are sufficiently large, then either the rule based on the equal sacrifice solution or the rule based on the split-the-surplus solution can support unarmed peace that would dominate the rule based on Nash bargaining that generates strictly positive equilibrium arming. Otherwise, each of the rules considered imply arming in equilibrium, and in this case the rule based on the equal sacrifice solution, which is least sensitive to the disagreement payoffs and thus implies the strongest norms against threats, emerges as the Pareto dominant rule.

Since our analysis is based on a very simple setting, it is natural for one to ask how the efficiency rankings extend to more general settings. Consider, for example, our assumption that initial resources are symmetrically distributed across the two agents. It turns out that this assumption is important only insofar as it allows us to abstract from outcomes where one of the two agents is resource constrained in the production of guns. That is to say, the equilibria in guns under peaceful settlement based on any of the rules of division considered as well as the equilibrium under war remain symmetric provided the resource constraint does not bind for either of the agents in his production of guns. As such, the efficiency ranking of the rules of division under settlement found above remain intact. Even when one agent is resource constrained under war, the finding that incentives to arm under peaceful settlement are lower than under war means that it is possible neither agent is resource constrained under settlement, in which case the efficiency rankings would continue to hold. Of course, if the initial distribution of the resource is sufficiently uneven, one of the agents could be resource constrained under one or more of the rules of division for peaceful settlement as well as under war. ${ }^{21}$ The implications for the efficiency ranking of the rules of division under settlement are not immediately obvious. ${ }^{22}$ Still, settlement would continue to dominate war for both agents since it involves less arming for the unconstrained agent; and, by our arguments above, war could not emerge in the one-period setting analyzed here.

This result would seem to form a strong basis for the classical liberal view. However, we caution the reader that it need not hold generally in a multi-period setting, under the reasonable assumption that commitments to the division of the resource in the future are not feasible. In particular, even if the two sides settle their dispute in the current period peacefully, they would have to negotiate a division of the resource in the future and that possibly requires additional arming. ${ }^{23}$ War, by contrast, could confer on the winner a strategic advantage in future disputes over the resource. One extreme possibility here, studied by Garfinkel and Syropoulos (2017b) while focusing on the split-the-surplus rule, is that victory in a war effectively wipes out the rival and thus eliminates the victor's need to arm in the future. In that dynamic setting, depending on the magnitude of the gains from trade, the destructiveness of war and the salience of the future, among other factors, war could emerge as the perfectly coalition-proof equilibrium. While under other parameterizations of the model peaceful settlement could Pareto dominate war, the arguments of the present paper in a one-period setting that rule out the profitability of unilateral deviations do not easily carry over to the multi-period setting. Nevertheless, results of the present paper suggest that the other bargaining protocols (namely, equal sacrifice and Nash bargaining) could be more conducive to peaceful settlement than the split-the-surplus solution in a multi-period setting.

\section{Acknowledgement}

We are indebted to Nejat Anbarci and Stergios Skaperdas, as well as the Associate Editor, Arzu Kibris, and three anonymous referees, for insightful comments and suggestions.

\section{A Appendix}

\section{Proof of Lemma 1:}

Part (1): Setting $\lambda^{i}=1 / 2$ and $\lambda^{i}=1$ in (4) gives $v^{i}\left(\frac{1}{2}\right)=2^{-\frac{\sigma-2}{\sigma-1}}$ and $v^{i}(1)=1$, respectively. The result then follows by comparing these payoffs. In particular, if $\sigma=2$, then $v^{i}\left(\frac{1}{2}\right)=1=v^{i}(1)$. In contrast, if $\sigma<2$ (resp., $\sigma>2$ ), then $v^{i}\left(\frac{1}{2}\right)>1\left(\right.$ resp., $\left.v^{i}\left(\frac{1}{2}\right)<1\right)$. 
Part (2): First note that differentiation of $v^{i}$ with respect to $\lambda^{i}$ (while, at the same time, allowing $\lambda^{j}$ to change since $\lambda^{j}=1-\lambda^{i}$ ) gives

$$
v_{\lambda^{i}}^{i} \equiv \frac{1}{\sigma v^{i} \lambda^{i} \lambda^{j}}\left[\sigma \lambda^{j}-\frac{\left(\lambda^{j}\right)^{\frac{\sigma-1}{\sigma}}}{\left(\lambda^{i}\right)^{\frac{\sigma-1}{\sigma}}+\left(\lambda^{j}\right)^{\frac{\sigma-1}{\sigma}}}\right] .
$$

Now define $\rho \equiv \lambda^{i} / \lambda^{j}$. Since $\lambda^{i}+\lambda^{j}=1$, we have $\lambda^{i}=\rho /(1+\rho)$ and $\lambda^{j}=1 /(1+\rho)$. Next observe that the (implicit) solutions to $v_{\lambda^{i}}^{i}=0$ and $v^{j}-u^{j}=0$ give $\lambda_{T}^{i}$ and $\tilde{\tilde{\lambda}}^{i}$, respectively. We may rewrite these conditions so that, in the former case, we have:

$$
\frac{1}{1-\lambda_{T}^{i}}=1+\rho=\sigma\left(1+\rho^{\frac{\sigma-1}{\sigma}}\right)
$$

Similarly, setting $G^{i}=G^{j}=G$ (which implies $u^{j}=\frac{1}{2}$ ) and using the definition of $v^{j}$ in the $v^{j}-u^{j}=0$ case we have

$$
\frac{1}{1-\tilde{\tilde{\lambda}}^{i}}=1+\rho=2\left(1+\rho^{\frac{\sigma-1}{\sigma}}\right)^{\frac{1}{\sigma-1}} .
$$

One can readily see now that both (20) and (21) have unique solutions in which $\rho>1$. Division of the expressions in the far right of (20) and (21) gives

$$
\frac{2}{\sigma}\left(1+\rho^{\frac{\sigma-1}{\sigma}}\right)^{-\frac{\sigma-2}{\sigma-1}}
$$

Since the left-hand sides of (20) and (21) involve $1+\rho$, the relationship of the above expression to 1 describes the relationship between $\lambda_{T}^{i}$ and $\tilde{\tilde{\lambda}}^{i}$. It is now easy to verify that $\sigma \gtreqless 2 \Rightarrow 1-\lambda_{T}^{i} \gtreqless 1-\tilde{\tilde{\lambda}}^{i}$ or, equivalently, $\sigma \gtreqless 2 \Rightarrow \lambda_{T}^{i} \lesseqgtr \tilde{\tilde{\lambda}}^{i} . \|$

\section{Proof of Lemma 2:}

To find the impact of an agent's arming on his share of the disputed resource, first we differentiate the equations in (12-14) to obtain:

$$
\begin{gathered}
\Phi_{\lambda^{i}}=-\frac{\Phi v_{\lambda^{i}}^{i}+v_{\lambda^{j}}^{j}}{v^{i}-u^{i}} \text { and } \Phi_{G^{i}}=\frac{\Phi u_{G^{i}}^{i}-u_{G^{i}}^{j}}{v^{i}-u^{i}} \\
\Psi_{\lambda^{i}}=\frac{\Psi v_{\lambda^{i}}^{i}+v_{\lambda^{j}}^{j}}{w^{i}-v^{i}} \text { and } \Psi_{G^{i}}=-\frac{\Psi w_{G^{i}}^{i}-w_{G^{i}}^{j}}{w^{i}-v^{i}} \\
\Omega_{\lambda^{i}}=\frac{-\Omega v_{\lambda i \lambda^{i}}^{i}+v_{\lambda j \lambda j}^{j}}{v_{\lambda^{i}}^{i}} .
\end{gathered}
$$

Differentiating the equations in (15-17) and using the implicit function theorem yield:

$$
\begin{gathered}
\partial \lambda_{s s}^{i} / \partial G^{i}=-\frac{\Phi_{G^{i}}}{\Phi_{\lambda^{i}}}=\frac{\Phi u_{G^{i}}^{i}-u_{G^{i}}^{j}}{\Phi v_{\lambda^{i}}^{i}+v_{\lambda^{j}}^{j}} \\
\partial \lambda_{e s}^{i} / \partial G^{i}=-\frac{\Psi_{G^{i}}}{\Psi_{\lambda^{i}}}=\frac{\Psi w_{G^{i}}^{i}-w_{G^{i}}^{j}}{\Psi v_{\lambda^{i}}^{i}+v_{\lambda^{j}}^{j}}
\end{gathered}
$$




$$
\partial \lambda_{n b}^{i} / \partial G^{i}=-\frac{\Phi_{G^{i}}}{\Phi_{\lambda^{i}}+\Omega_{\lambda^{i}}}=\frac{\Phi u_{G^{i}}^{i}-u_{G^{i}}^{j}}{\Phi v_{\lambda^{i}}^{i}+v_{\lambda^{j}}^{j}-\left(v^{i}-u^{i}\right)\left(\frac{-\Omega v_{\lambda i \lambda^{i}}^{i}+v_{\lambda_{\lambda} j}^{j}}{v_{\lambda^{i}}^{i}}\right)} .
$$

Since the signs and magnitudes of the expressions in (22-24) play key roles in the determination of $\partial \lambda_{h}^{i} / \partial G^{i}$ shown in (25-27), it pays to investigate them now. First, recalling that $u^{i} \equiv \phi^{i}(i=1,2)$ and using (5), we have $u_{G^{i}}^{i}=\phi_{G^{i}}^{i}>0$ and $u_{G^{i}}^{j}=-\phi_{G^{i}}^{i}<0$ as well as $v^{i}>u^{i}$, which from (22) imply $\Phi_{G^{i}}>0$. Second, we have $v_{\lambda^{i}}^{i}>0$ for $\lambda^{i} \in\left[\frac{1}{2}, \lambda_{T}^{i}\right)(i=1,2)$. As such, under assumption (11) which implies $\lambda^{i}=\lambda^{j}=\frac{1}{2}, v_{\lambda^{i}}^{i}>0$ holds in equilibrium. Then, it follows from (22) that $\left.\Phi_{\lambda^{i}}\right|_{\lambda^{i}=1 / 2}<0$ and, thus, from (25), that $\partial \lambda_{s s}^{i} / \partial G^{i}>0$ holds.

The above discussion also sheds light on the sign of $\partial \lambda_{n b}^{i} / \partial G^{i}$ and its ranking relative to $\partial \lambda_{s s}^{i} / \partial G^{i}$. As noted in the text and as can be confirmed by differentiating $v_{\lambda^{i}}^{i}$ in (19) to find

$$
v_{\lambda^{i} \lambda^{i}}^{i}=-\frac{1}{v^{i}}\left\{\frac{\left(\lambda^{j}\right)^{-\frac{\sigma+1}{\sigma}}\left[\left(\lambda^{i}\right)^{\frac{\sigma-1}{\sigma}}+(\sigma-1)\left(\lambda^{j}\right)^{\frac{\sigma-1}{\sigma}}\right]}{\left(\lambda^{i}\right)^{2} \sigma^{2}\left[\left(\lambda^{i}\right)^{\frac{\sigma-1}{\sigma}}+\left(\lambda^{j}\right)^{\frac{\sigma-1}{\sigma}}\right]^{2}}\right\}<0,
$$

$v^{i}$ is strictly concave in $\lambda^{i}$ for both players provided $\sigma>1$. In addition, from (14) and the definition of $\lambda_{T}^{i}$, one can then see $\Omega \equiv \Omega\left(\lambda^{i}\right)<0$ for $\lambda^{i} \in\left[\frac{1}{2}, \lambda_{T}^{i}\right)$. Thus, the last term in the denominator of the far right expression in (27) is positive. This point and the analysis in the previous paragraph imply $\partial \lambda_{s s}^{i} / \partial G^{i}>\partial \lambda_{n b}^{i} / \partial G^{i}>0$, the last inequality shown in Lemma 2 . In words, the concavity of payoff functions in shares (which enters the determination of distributive shares under $n b$ but not under ss) renders $\lambda_{n b}^{i}$ less sensitive to changes in guns than $\lambda_{s s}^{i}$ under the conditions of the lemma.

Turning to $\partial \lambda_{e s}^{i} / \partial G^{i}$, the definition of the ideal payoffs in (10) reveals that changes in arming will influence these payoffs only in cases where $w^{i}=v^{i}\left(\tilde{\tilde{\lambda}}^{i}\right.$ ) (which as discussed earlier depends on the value of $\sigma$, an inverse proxy of the gains from trade). Focusing on this case, (8) implies $w_{G^{i}}^{i}=\tilde{v}_{\lambda^{i}}^{i}\left(\partial \tilde{\tilde{\lambda}}^{i} / \partial G^{i}\right)=-\tilde{\tilde{v}}_{\lambda^{i}}^{i}\left(\partial \tilde{\lambda}^{j} / \partial G^{i}\right)$ since $\tilde{\tilde{\lambda}}^{i}=1-\tilde{\lambda}^{j}$, for $i \neq j=1$, 2. Equation (8) with assumption (11) further implies $\partial \tilde{\lambda}^{j} / \partial G^{i}=u_{G^{i}}^{j} / v_{\lambda^{j}}^{j}$. Repeating this argument for changes in $w^{j}$ gives

$$
\begin{gathered}
w_{G^{i}}^{i}=\frac{\tilde{\tilde{v}}_{\lambda^{i}}^{i}}{\tilde{v}_{\lambda^{j}}^{j}}\left(-u_{G^{i}}^{j}\right) \\
w_{G^{i}}^{j}=\frac{\tilde{\tilde{v}}_{\lambda^{j}}^{j}}{\tilde{v}_{\lambda^{i}}^{i}}\left(-u_{G^{i}}^{i}\right) .
\end{gathered}
$$

Thus, provided $\lambda^{i} \in\left[\frac{1}{2}, \lambda_{T}^{i}\right)$ for both players, $w_{G^{i}}^{i}>0$ and $w_{G^{i}}^{j}<0$ hold. To proceed further, we must make more intensive use of our symmetry assumption. Accordingly, we focus on the case of equal arming (i.e. $G^{i}$ $=G$ for $i=1,2)$, which implies, among other things, $u_{G^{i}}^{j}=-u_{G^{i}}^{i} v_{\lambda^{j}}^{j}=v_{\lambda^{i}}^{i} v_{\lambda^{j} \lambda^{j}}^{j}=v_{\lambda^{i} \lambda^{i}}^{i}$ and enables us to rewrite $\tilde{v}_{\lambda^{i}}^{i} / \tilde{v}_{\lambda^{j}}^{j}=\tilde{v}_{\lambda^{j}}^{j} / \tilde{v}_{\lambda^{i}}^{i}$ as $\tilde{v}_{\lambda^{i}}^{i} / \tilde{v}_{\lambda^{i}}^{i}$ (since $\tilde{v}_{\lambda^{j}}^{j}=\tilde{v}_{\lambda^{i}}^{i}$. But, the strict concavity of $v^{i}$ in $\lambda^{i}$ implies $\tilde{v}_{\lambda^{i}}^{i} / \tilde{v}_{\lambda^{i}}^{i}<1$; thus, $w_{G^{i}}^{i}=-w_{G^{i}}^{j}<u_{G^{i}}^{i}$. Since as can easily be confirmed $\Phi=\Psi=-\Omega=1$ under symmetry, a comparison of (25) and (26) shows that $\partial \lambda_{s s}^{i} / \partial G^{i}>\partial \lambda_{e s}^{i} / \partial G^{i}>0$. Thus, the division under $e s$ is less sensitive than division under ss to an agent's arming, due to the indirect (and weaker) dependence of the ideal payoffs on the threat point.

What we do not know yet is how $\partial \lambda_{e s}^{i} / \partial G^{i}$ compares with $\partial \lambda_{n b}^{i} / \partial G^{i}$. Such a comparison requires a considerable amount of additional work. Applying the implications of symmetry to (26) and (27), we can rewrite these expressions, after simplification, as

$$
\begin{gathered}
\partial \lambda_{e s}^{i} / \partial G^{i}=\frac{\left(\tilde{\tilde{v}}_{\lambda^{i}}^{i} / \tilde{v}_{\lambda^{j}}^{j}\right) u_{G^{i}}^{i}}{v_{\lambda^{i}}^{i}}=\frac{u_{G^{i}}^{i} / v_{\lambda^{i}}^{i}}{\tilde{v}_{\lambda^{j}}^{j} / \tilde{v}_{\lambda^{i}}^{i}} \\
\partial \lambda_{n b}^{i} / \partial G^{i}=\frac{u_{G^{i}}^{i}}{v_{\lambda^{i}}^{i}-\left(v^{i}-u^{i}\right)\left(v_{\lambda^{i} \lambda^{i}}^{i} / v_{\lambda^{i}}^{i}\right)}=\frac{u_{G^{i}}^{i} / v_{\lambda^{i}}^{i}}{1-\left(1-u^{i} / v^{i}\right)\left[v^{i} v_{\lambda^{i} \lambda^{i}}^{i} /\left(v_{\lambda^{i}}^{i}\right)^{2}\right]} .
\end{gathered}
$$


Note that $\tilde{v}_{\lambda^{j}}^{j} / \tilde{\tilde{v}}_{\lambda^{i}}^{i}$ is the slope of the PPF at $w^{i}-$ or, in the context of Figure 2(B), the slope of the PPF at point $E$ (but with $G^{1}=G^{2}$ ) or at point $E$ in Figure 1 .

Now, using the transformations $\lambda^{i}=\rho /(1+\rho)$ and $\lambda^{j}=1 /(1+\rho)$ in the definitions of $\tilde{v}_{\lambda^{j}}^{j}$ and $\tilde{\tilde{v}}_{\lambda^{i}}^{i}$ we may rewrite $\tilde{v}_{\lambda^{j}}^{j} / \tilde{v}_{\lambda^{i}}^{i}$ in $(31)$ as

$$
\tilde{v}_{\lambda^{j}}^{j} / \tilde{\tilde{v}}_{\lambda^{i}}^{i}=\frac{\rho^{\frac{1}{\sigma-1}} \sigma-\frac{1+\rho}{1+\rho^{\frac{\sigma-1}{\sigma}}}}{\sigma-\frac{1+\rho}{1+\rho^{\frac{\sigma-1}{\sigma}}}}>1 \text {, since } \rho>1 \text { at } v^{j}-u^{j}=0 \text {. }
$$

Turning to (32), $u^{i} / v^{i}=2^{-\frac{1}{\sigma-1}}\left(\right.$ since $u^{i}=1 / 2$ and $\left.v^{i}=2^{-\frac{\sigma-2}{\sigma-1}}\right)$ and $-v^{i} v_{\lambda^{i} \lambda^{i}}^{i} /\left(v_{\lambda^{i}}^{i}\right)^{2}=\sigma /(\sigma-1)^{2}$ in the denominator. Upon substitution of these expressions in (31) and (32), we arrive at

$$
\Lambda(\rho, \sigma) \equiv \frac{\partial \lambda_{e s}^{i} / \partial G^{i}}{\partial \lambda_{n b}^{i} / \partial G^{i}}=\left[\frac{\sigma-\frac{1+\rho}{1+\rho^{\frac{\sigma-1}{\sigma}}}}{\rho^{\frac{1}{\sigma-1}} \sigma-\frac{1+\rho}{1+\rho^{\frac{\sigma-1}{\sigma}}}}\right]\left[1+\frac{\sigma\left(1-2^{-\frac{1}{\sigma-1}}\right)}{(\sigma-1)^{2}}\right]>0
$$

Since the expression inside the first set of brackets is smaller than 1 while the expression in the second set of brackets is larger than 1, it is unclear how es and $n b$ compare in the sensitivities of the shares they give rise to. Nevertheless, we can resolve this ambiguity constructively, with the use of numerical methods that exhaust all possibilities.

To this end, note from (21), that the value of $\rho$ that solves $v^{j}-u^{j}=0$ coincides with the solution to $1+\rho=$ $2\left(1+\rho^{\frac{\sigma-1}{\sigma}}\right)^{\frac{1}{\sigma-1}}$. Denote this solution as $\rho_{0}(\sigma)$. It is straightforward to show that $\rho_{0}$ is unique and that $d \rho_{0} / d \sigma<0$. We calculated $\rho_{0}(\sigma)$ for values of $\sigma \in(2,1000)$ using a grid of 0.001 . The calculations relied on Newton's method. Substituting each value of $\rho_{0}$ into $\Lambda(\cdot)$ and evaluating the resulting expression confirms that, indeed, $\Lambda\left(\rho_{0}(\sigma)\right.$, $\sigma)<1$ for all relevant values of $\sigma$. In short, $\partial \lambda_{n b}^{i} / \partial G^{i}>\partial \lambda_{e s}^{i} / \partial G^{i}>0$, which completes the proof. $\|$

\section{Proof of Proposition 1:}

With our maintained focus on symmetric equilibria that imply $\lambda_{h}^{i}=1 / 2$ for all $h$, we derive the following expressions for the relevant terms appearing in (18): $v^{i}=2^{-\frac{\sigma-2}{\sigma-1}}, v_{\lambda^{i}}^{i}=2^{\frac{1}{\sigma-1}} \frac{(\sigma-1)}{\sigma}$, and $\bar{X}=2(R-G)$. Substituting these relationships in (18) and simplifying the resulting expression gives

$$
\frac{\partial V_{h}^{i}}{\partial G^{i}}=2^{-\frac{\sigma-2}{\sigma-1}}\left[\frac{4(\sigma-1)(R-G)}{\sigma}\left(\frac{\partial \lambda_{h}^{i}}{\partial G^{i}}\right)-1\right]
$$

The above expression makes two things clear. First, a corner solution with $G_{h}=0$ arises if $\partial \lambda_{h}^{i} / \partial G^{i}=0$. The intuition for this result is that, if an increase in an agent's arming does not raise his share of the contested resource, then his marginal benefit will vanish and it would be pointless to incur the cost of producing any guns. Second, if $\partial \lambda_{h}^{i} / \partial G^{i}>0$, then the marginal benefit of producing guns will be positive and, as long as this benefit is not dominated by the associated marginal cost, a positive quantity of arms will be observed. What's more, because an agent's marginal benefit is increasing in the sensitivity of his share to his arming, the bargaining solutions can be ranked in terms of their relative sensitivities.

Noting that there is no closed-form solution to $\partial \lambda_{e s}^{i} / \partial G^{i}$ when $\partial \lambda_{e s}^{i} / \partial G^{i}>0$, we apply the implications of symmetry to $\partial \lambda_{h}^{i} / \partial G^{i}$ in (25-27) for $h \in\{s s, n b\}$ using (22-24) to obtain

$$
\begin{aligned}
& \partial \lambda_{s S}^{i} / \partial G^{i}=\frac{1}{4 G}\left[2^{\frac{1}{\sigma-1} \frac{(\sigma-1)}{\sigma}}\right]^{-1}=\frac{1}{4 G A}>0 \\
& \partial \lambda_{n b}^{i} / \partial G^{i}=\frac{1}{4 G}\left[2^{\frac{1}{\sigma-1}} \frac{(\sigma-1)}{\sigma}+\frac{2^{\frac{1}{\sigma-1}}-1}{\sigma-1}\right]^{-1}=\frac{1}{4 G(A+B)}>0,
\end{aligned}
$$

where $A \equiv 2^{\frac{1}{\sigma-1}} \frac{(\sigma-1)}{\sigma}$ and $B \equiv \frac{2^{\frac{1}{\sigma-1}}-1}{\sigma-1}$, as defined in the proposition. Substituting the above expressions in (34) and solving for $G$ gives the expressions for the unique $G_{s S}$ and $G_{n b}$ in the interior of the strategy space noted in the proposition.

Let us now take a closer look at each solution concept. Starting with ss, for space reasons and as noted in the text, we just point out that technical details on the $G_{s S}=0$ case for $\sigma \in\left[1 \frac{1}{2}, 2\right]$ are contained Garfinkel and Syropoulos (2017b). The intuition was explained in the text. 
Turning to $n b$, the solution is always in the interior of the strategy space because an agent always has an incentive to operate on the interior (and downward-sloping part) of the PPF, which requires some arming.

Lastly, with regards to the es solution, we note that whether $G_{e s}$ is zero or positive hinges on the possible dependence (or not) of the agents' ideal payoffs on their disagreement payoffs. But, as noted in part (2) of Lemma 1, this dependence (or, its absence) hinges on whether $\sigma<2$ (or not). $\|$

\section{Notes}

1 While Oneal and Russett (1997 and 1999), for example, present evidence that economic interdependence between two nations is negatively related to the likelihood of war breaking out between them, Barbieri (1996 and 2002) finds no significant relation between trade and war. See Copeland (2015) for a survey of alternative views and empirical evidence regarding trade and war found in the international relations literature.

2 See, for example, Skaperdas and Syropoulos (2001), Garfinkel, Skaperdas, and Syropoulos (2015), and Garfinkel and Syropoulos (2017a). Also, see Bonfatti and O'Rourke (2017) who study how increasing trade dependence for two adversarial countries with the rest of the world can influence the likelihood of a preemptive war.

3 See, for example, Anbarci, Skaperdas, and Syropoulos (2002). Also, see Garfinkel, McBride, and Skaperdas (2012), who analyze how norms and the more formal institutions of governance that help define and enforce property rights matter jointly in determining the possible gains from settlement.

4 We follow Anbarci, Skaperdas, and Syropoulos (2002) in studying the split-the-surplus and equal sacrifice solutions; but, whereas we also consider the Nash bargaining solution, they consider the Kalai-Smorodinsky solution.

5 Martin, Mayer, and Thoenig (2008) explore how trade opportunities between the two contending countries alone and between each country and others can influence the effectiveness of bargaining to avoid war under uncertainty, but they do not consider explicitly the resource costs involved.

6 In Garfinkel and Syropoulos (2017b), we study in a dynamic setting the countries' choices of both arming and whether to settle peacefully or go to war. As discussed in some detail below, we find that, war can emerge as the perfectly coalition-proof equilibrium, despite its effect to preclude mutually advantageous trade. The difference in results is due to commitment problems that have been analyzed by others in different dynamic settings without trade (e.g. Garfinkel \& Skaperdas, 2000; McBride \& Skaperdas, 2014). While the analysis in the oneperiod setting of the present paper abstracts from these problems, it does bring to light the importance of considering alternative rules of division in assessing the prospects of peace in the presence of economic interdependence when commitment problems are relevant.

7 To highlight the salience of trade, we abstract from war's destructive effects. This assumption and our assumption that war precludes trade could be relaxed, but at a cost of added analytical complexity.

8 We will describe the three bargaining protocols shortly. Until then, we omit the subscript associated with division $h$ to avoid notational clutter.

9 Formally, the assumed structure of production and trade is consistent with the Armington (1969) model. The analysis could be extended to allow for the possibility of Ricardian type of trade (where each agent $i=1,2$ can produce both intermediate goods $j=1,2$, but has a comparative advantage in one, say good $j=i$ ) without changing our results qualitatively. By the same token, our assumption that all of the residual resource $R^{i}-G^{i}$ held by each agent is subject to dispute could be relaxed to allow for some (imperfect) degree of security. We have confirmed that, if the structure of comparative advantage and the degree of resource insecurity are symmetric, the analysis could be extended in these two ways simultaneously without changing the results qualitatively.

10 The restriction that $\sigma>1$ is imposed to ensure that output under autarky (using one input only) is well-defined and strictly positive. Otherwise, the threat-point payoff would equal zero and there would be no arming under any of the bargaining protocols considered.

11 The expression inside the square brackets is the elasticity of butter production with respect to agent $i$ 's input.

12 In Appendix A (see the proof of Lemma 2), we prove $v_{\lambda^{i} \lambda^{i}}<0$. Garfinkel and Syropoulos (2017b) establish these properties hold under more general circumstances, including the presence of "iceberg" type trade costs due to geographic trade barriers.

13 See Tullock (1980), Hirshleifer (1989), and Skaperdas (1996) for analyses and discussions of this form.

14 More precisely, the equilibrium in arming under war is symmetric provided that initial resources, $R^{1}$ and $R^{2}$, are not too unevenly distributed across agents. Letting $\bar{R}=R^{1}+R^{2}$, this condition can be written as $R^{i} \in\left[\frac{1}{4} \bar{R}, \frac{3}{4} \bar{R}\right)$ for $i=1,2$.

15 Note that, when $G^{1}>G^{2}\left(G^{1}<G^{2}\right)$, the most southwest point of each bargaining set (given $\sigma>1$ ) shown in the figure moves right (left) along the $u^{1}+u^{2}=1$ frontier.

16 Note that this claim does not mean that either agent prefers war in this case.

17 Also, keep in mind that the scale for panel (B) is larger than that for panel (A) to allow for a clearer illustration of the differences in the solutions $h \in\{s s, e s, n b\}$ when $\sigma>2$.

18 Since the no-arming equilibrium Pareto dominates the arming equilibrium, when $\sigma \in\left(\frac{3}{2}, 2\right)$ such that both are possible, the two agents in their negotiations would presumably communicate and coordinate their actions. Put differently, the arming equilibrium in such cases is not perfectly coalition-proof, and thus can be ruled out in favor of the no-arming equilibrium. See Garfinkel and Syropoulos (2017b) for further discussion of this point.

19 In particular, since arming induced by the split-the-surplus rule of division is greater than that induced by the other rules of division, one need only confirm that $G_{s S}>G_{c}$ or equivalently (using the solution for $G_{s S}>0$ shown in Proposition 2 and the solution for $G_{c}$ implied by (7) in the case of symmetry) that $1<2^{\frac{1}{\sigma-1}}$, which holds for $\sigma \in(1, \infty)$.

20 Note that the Kalai-Smorodinsky $(k s)$ solution is defined implicitly by the condition $\Phi\left(\lambda_{k s}^{i}\right)=\Psi\left(\lambda_{k s}^{i}\right)$. Although we do not study this solution in detail here, we note that the impact of an agent's guns on the share of the contested resource under the $k s$ solution is a weighted sum of the marginal effects of his guns on the shares under the $e s$ and ss solutions; consequently, $G_{e s}<G_{k s}<G_{s s}$ for all $\sigma \in(2, \infty)$. In addition, numerical analysis suggests that for all $\sigma \in(1, \infty)$ the $k s$ solution implies a lower level of arming than the $n b$ solution.

21 Given the ranking of the incentives for agents to arm under $s s, n b$ and $e s$ (assuming $\sigma>2$ ), successive shifts in the initial distribution of resources away from one agent and towards the other would render the resource constraint binding (for one country) more quickly under $s s$, then $n b$ and finally under es.

22 Interestingly, numerical analysis indicates that, if one agent $i$ is sufficiently larger such that agent $j$ is resource constrained under each of the bargaining solutions, agent $j$ 's preference ordering over the three rules remains intact; by contrast, the unconstrained agent could prefer splitting the surplus to Nash bargaining and prefer Nash bargaining to equal sacrifice. Thus, it is possible that no rule is Pareto efficient. 
23 See Fearon (1995) and Powell (2006), as well as Garfinkel and Skaperdas (2000) and McBride and Skaperdas (2014) for discussions on this point.

\section{References}

Anbarci, N., Skaperdas, S., \& Syropoulos, C. (2002). Comparing bargaining solutions in the shadow of conflict: How norms against threats can have real effects. Journal of Economic Theory, 106(1), 1-16.

Armington, P. S. (1969). A theory of demand for products distinguished by place of production. International Monetary Fund Staff Papers, 16(1), 159-176.

Barbieri, K. (1996). Economic interdependence: A path to peace or a source of interstate conflict? Journal of Peace Research, 33(1), 29-49.

Barbieri, K. (2002). The Liberal Illusion: Does Trade Promote Peace? Ann Arbor: The University of Michigan Press.

Bernheim, B. D., Peleg, B., \& Whinston, M. D. (1987). Coalition-proof nash equilibria: I. Concepts. Journal of Economic Theory, 42(1), 1-12.

Bonfatti, R. \& O’Rourke, K. H. (2017). Growth, import dependence and war. Economic Journal. http://onlinelibrary.wiley.com/doi/10.1111/ecoj.12511/full.

Copeland, D. C. (2015). Economic Interdepence and War. Princeton: Princeton University Press.

Fearon, J. (1995). Rationalist explanations for war. International Organization, 49(3), 379-414.

Garfinkel, M. R., \& Skaperdas, S. (2000). Conflict without misperceptions or incomplete information: How the future matters. Journal of Conflict Resolution, 44(6), 793-807.

Garfinkel, M. R., \& Syropoulos, C. (2017a). Trading with the enemy. Unpublished paper.

Garfinkel, M. R., \& Syropoulos, C. (2017b). On trade and the stability of (armed) peace. Unpublished paper.

Garfinkel, M. R., McBride, M., \& Skaperdas, S. (2012). Covernance and norms as determinants of arming. Revue d'Economie Politique, 122(2), 197-212.

Garfinkel, M. R., Skaperdas, S., \& Syropoulos, C. (2015). Trade and insecure resources. Journal of International Economics, 95(1), 98-114.

Glick, R., \& Taylor, A. M. (2010) Collateral damage: Trade disruption and the economic impact of war. Review of Economics and Statistics, 92(1), 102-127.

Hirshleifer, J. (1989). Conflict and rent-seeking success functions: Ratio vs. difference models of relative success. Public Choice, 63(2), 101-112.

Martin, P., Mayer, T., \& Thoenig, M. (2008). Make trade not war. Review of Economic Studies, 75(3), 865-900.

McBride, M., \& Skaperdas, S. (2014). Conflict, settlement and the shadow of the future. Journal of Economic Behavior and Organization, 105, 75-89.

Oneal, J. R., \& Russett, B. M. (1997). The classical liberals were right: Democracy, interdependence and conflict, 1950-1985. International Studies Quarterly, 41(2), 267-293.

Oneal, J. R., \& Russett, B. M. (1999). The Kantian peace: The Pacific benefits of democracy, interdependence and international organizations, 1855-1992. World Politics, 52(1), 1-37.

Polachek, S. W. (1980). Conflict and trade. Journal of Conflict Resolution, 24, 55-78.

Powell, R. (2006). War as a commitment problem. International Organization, 60(1), 169-203.

Skaperdas, S. (1996). Contest success functions. Economic Theory, 7(2), 283-290.

Skaperdas, S., \& Syropoulos, C. (2001). “Guns, butter, and openness: On the relationship between security and trade. American Economic Review: Papers and Proceedings, $91(2), 353-357$.

Tullock, G. (1980). Efficient rent seeking. In J. M. Buchanan, R. D. Tollison \& G. Tullock (Eds.), Toward a theory of the rent seeking society (pp. 3-15). College Station: Texas A\&M University Press. 